\title{
Filigrane
}

Écoutes psychothérapiques

\section{Le problème de l'espace en psychologie du sportif. L'espace thymique comme foyer du lien " soi-monde-autrui "}

\section{Brigitte Leroy-Viemon et Félicia Moscato}

Volume 17, numéro 2, automne 2008

L’avenir du clinicien II

URI : https://id.erudit.org/iderudit/019427ar

DOI : https://doi.org/10.7202/019427ar

Aller au sommaire du numéro

Éditeur(s)

Revue Santé mentale au Québec

ISSN

1192-1412 (imprimé)

1911-4656 (numérique)

Découvrir la revue

Citer cet article

Leroy-Viemon, B. \& Moscato, F. (2008). Le problème de l'espace en psychologie du sportif. L'espace thymique comme foyer du lien " soi-monde-autrui ».

Filigrane, 17(2), 174-194. https://doi.org/10.7202/019427ar
Résumé de l'article

Il est une qualité d'espace, l'espace thymique (Binswanger, 1932), qui mérite d'être davantage connue en psychologie clinique car elle re-crée, chez l'homme, le sentiment " d'exister avec autrui », optimalisant, au final, la prise en charge psychothérapeutique. 


\title{
Le problème de l'espace en psychologie du sportif. L'espace thymique comme foyer du lien «soi-monde-autrui »
}

\author{
brigitte leroy-viemon \\ félicia moscato
}

\begin{abstract}
Il est une qualité d'espace, l'espace thymique (Binswanger, 1932), qui mérite d'être davantage connue en psychologie clinique car elle re-crée, chez l'homme, le sentiment «d'exister avec autrui », optimalisant, au final, la prise en charge psychothérapeutique.
\end{abstract}

A près avoir situé le concept d'espace thymique par rapport aux connaissances psychanalytiques concernant la relation d'objet et phénoménologiques concernant la relation homme-monde, les auteurs en illustrent la valeur opératoire à partir d'une expérience de terrain menée dans un atelier d'expression par le corps et par la voix avec des personnes désorientées après un traumatisme crânien.

Enfin, ils formulent des pistes de travail qui s'avèrent pertinentes pour la prise en charge du sujet sportif confronté aux problèmes récurrents du stress, du manque de confiance en soi, de l'absence de cohésion d'équipe, de la relation conflictuelle à l'entraîneur... En somme, toute la variété des problèmes psychologiques qui interrogent la dimension pré-psychique du rapport «soi-monde-autrui » et notamment la disposition thymique à orienter ses investissements pulsionnels.

\section{Où il est question d'espaces}

Qu'il s'agisse de l'intervention psychologique en milieu sportif ou de l'intervention psychologique dans le champ du handicap, il nous semble que le problème de l'espace est trop souvent abordé du seul point de vue de l'espace orienté, c'està-dire d'un espace conditionné de multiples manières par le placement et la position du corps propre, par le mouvement, par les sens à distance (perceptions visuelle et auditive). Or, les recherches menées par notre équipe ${ }^{1}$ sur les dynamiques pré psychiques et psychiques de «l'homme en mouvement» nous enjoignent à considérer ce dernier — et a fortiori le sujet sportif — du point de vue d'une autre qualité d'espace: l'espace thymique (Binswanger, 1932).

Ni géométrique, ni pragmatique, ni même finalisé, l'espace thymique introduit l'homme à une compétence particulière: une manière autre d'être au monde, plus 
intérieure, plus sensible, habitée, sereine, unifiée, accueillante, éveillée aux autres et à soi. Cette disposition s'avère décisive du point de vue de la capacité du sportif à «se trouver» et à «trouver autrui » par le «sentant» plutôt que par le «voyant». La visée de cet article est donc de proposer à l'appréciation des psychologues intervenant dans le milieu sportif, ce concept d'espace thymique issu de la compréhension phénoménologique et intégrative de l'homme (Binswanger, 1932). Nous pensons que mieux connaître cette qualité intérieure devrait aider le psychologue à mieux aborder les problématiques récurrentes du stress, du manque de confiance en soi, de l'absence de cohésion d'équipe, de la relation conflictuelle à l'entraineur [...]; des problématiques psychologiques qui interrogent:

«les sources des pulsions freudiennes jusqu'en deçà de l'érotisation qualifiée à laquelle Freud s'était arrêté» (Schotte, 1990, 11)

région de l'âme où la disposition thymique oriente les investissements pulsionnels, ajoutons-nous, étayant notre propos sur la compréhension phénoménologique de «l'être homme».

Nous commencerons par situer théoriquement le concept d'espace thymique par rapport aux connaissances psychanalytiques (la relation d'objet dans son sens large) et phénoménologiques (la relation homme-monde) concernant les dynamiques du lien à autrui.

Puis nous exposerons l'exemple d'une expérience de terrain menée dans un atelier d'expression par le corps et par la voix avec des personnes ayant subi un traumatisme crânien pour illustrer la valeur opératoire de l'espace thymique. Le choix de cette population, plutôt que celui d'une population de sportifs, est justifié par le fait que les effets du dispositif psychothérapeutique proposé y sont plus marqués; l'exposé devrait en tirer une plus grande portée didactique.

Concrètement, l'expérience menée avec les patients traumatisés crâniens montrera qu'un travail mené sur la dimension thymique de l'espace, peut re-créer l'unité d'un «sentiment d'exister avec autrui» que le trauma puis le handicap avaient disloqué. Les vertus créatrices du «séjour dans l'espace thymique» nous intéresseront d'autant plus que leurs effets peuvent perdurer en dehors de l'atelier, conférant, à l'homme handicapé, une fermeté nouvelle et très personnelle: celle d'un tonus moins «musculaire» que «présentiel». Par cette «création de tenue», la personne (traumatisée crânienne ou sportive) se trouve en disposition d'établir un lien avec autrui selon un esprit de coopération ${ }^{2}$ qui se substitue au comportement de retrait sur soi (inhibition) ou d'agressivité relationnelle (conscient et inconscient) ordinairement constatés.

\section{Aux confins de la relation d'objet psychanalytique}

En psychanalyse, le modèle du lien à autrui auquel nous nous référons est celui de la relation d'objet, inféré de la théorie freudienne des pulsions. De ce point de 
vue, la pérennité du lien est garantie par un incessant travail de mise en représentation (imaginaire et symbolique) par lequel le Moi et ses instances régulent le rapport de l'homme à son environnement. Incessante est cette activité psychique car l'imprévu (le trauma pour Freud, le Réel pour Lacan) pointe toujours son nez à l'angle de la rue... Et heureusement ! car ce Réel, s'il se présente dans une juste proportion, va stimuler l'activité psychique du sujet et permettre l'élaboration de montages représentationnels plus complexes, mieux adaptés à parer l'excitation psychique (l'angoisse) que le Réel en approche suscite toujours.

L'expérience psychopathologique montre que, face au Réel, l'activité représentationnelle n'est pas toujours en mesure de produire des montages opérants. La psychanalyse de ces situations extrêmes a permis à Freud et à ses successeurs d'éclairer les dynamiques du lien dit «normopathe» (non pathologique). Et d'inférer que la vie psychique est organisée par deux registres de signes hétérogènes: le registre proto représentationnel du processus originaire et ses pictogrammes (Aulagnier, 1975) et le registre représentationnel des processus primaires et secondaires et leurs représentations d'images et de mots. Il est acquis qu'originaire et représentationnel co-appréhendent simultanément le Réel (Aulagnier, 1975) tout au long de la vie humaine.

Contemporaine de Freud, l'Ecole Hongroise de Psychanalyse, autour de Ferenczi son fondateur, de Mélanie Klein, Léopold Szondi, Imhre Hermann, Michaël Balint et René Spitz pour ne citer que les plus fameux, s'est plus particulièrement intéressée aux commencements de la vie psychique: à ses formes d'organisation pré oedipiennes, pré fantasmatiques, pré narcissiques, pré objectales. Dans la continuité de ces travaux, les avancées de Winnicott vont constituer tout un pan de connaissances qui éclairent désormais (en psychanalyse) les confins de la relation d'objet en termes de: motricité primitive, spontanéité, créativité, capacité de sollicitude, aire transitionnelle d'expérience, mère suffisamment bonne, etc.

L'appui conceptuel de la phénoménologie permet d'approfondir encore, selon nous, la compréhension de ce qui fait consister le lien à autrui, aux marges de la vie psychique, là où le réel tient lieu de butée théorique pour la psychanalyse. $\mathrm{La}$ méthode phénoménologique appréhende, en effet, un niveau d'activité pré psychique, très corporel, très aesthésique, issu du sens oral (Tellenbach, 1968): le Sentir (Straus, 1935). Sentir et originaire désignent (dans leur discipline respective) des réalités qui, pour nous, s'inscrivent dans une continuité cohérente car certaines formes issues du Sentir s'expriment en proto-représentations originaires tandis que d'autres s'expriment uniquement par le mouvement, hors représentation (Moraguès, 2003).

\section{Sentir, stimmung et espace thymique en phénoménologie}

Notre conception, étayée par les travaux majeurs que nous avons cités, est que le Sentir et le représentationnel ont en partage la mission d'assurer la continuité de la vie psychique de l'homme; par conséquent, de métaboliser le Réel qui met en péril 
cette continuité (Leroy-Viémon, Trouillet et Bruère-Dawson, 2006). Le Sentir y participe, selon son mode propre, par la mise en rythme des fragments pictogrammiques (sortes de formes discontinues) qui se créent dans la rencontre hommemonde (sphère de l'intersubjectivité). Cette mise en rythme fabrique, hors représentation, une continuité existentielle qui se signale au sujet sous la forme d'une Stimmung, d'un sentiment «de se trouver» : un sentiment d'existence (Heidegger, 1941, 134). La manière, très personnelle, dont s'agencent ces productions discontinues, c'est-à-dire dont s'harmonisent, se mettent en rythme les fragments pictogrammiques, constitue le style présentiel d'une personne (Maldiney, 1973).

Dans son article sur les formes du spatial, Straus montre que l'espace thymique (il le nomme espace pathique ou présentiel) peut être appréhendé par le mouvement, notamment dansé (Straus, 1992). L'espace y est alors déterminé par l'ampleur, la hauteur, la profondeur du mouvement propre du danseur. Selon cet auteur, un tel mouvement déploie le temps et fabrique un horizon qui se déplace toujours par lui (Straus, 1992) : par ce mouvement, l'homme fonde un monde pour soi ; un monde ouvert à l'accueil du Réel sous le jour de l'altérité.

On comprendra qu'un tel mouvement ne se réduit pas à une simple action musculaire du corps mais qu'il provient d'une source plus «intérieure». En effet, thymique qui vient du grec thymos, désigne le centre à partir duquel quelque chose comme une Stimmung peut advenir et disposer l'homme présent à son projet. Thymique veut donc dire «disposé (orienté) par une Stimmung » qui a son origine dans le thymos en tant que «cœur» de la possibilisation spatiale et guide de la conduite (Gros, 1998). Une telle Stimmung, à l'exemple de celle qui guide le mouvement propre du danseur et que l'on peut traduire par «humeur» ou par «tonalité affective de base», se caractérise par l'amplitude: l'épanouissement ou le serrement de cœur; la diastole-systole de tout contact avec le monde. Plus simplement: qui n'a pas vécu l'expérience de trouver sa direction non pas à partir de la qualité «orientée» de l'espace mais à partir de sa qualité thymique ? Qui n'a pas vécu l'expérience «d'y aller» parce que «le cœur lui en dit» ou, à l'inverse, «de se garder d'y aller» tellement le «cœur n'y est pas»? Qui se s'est pas senti, par cette expérience même, le cœur gonflé de confiance et d'espoir ou à l'inverse, faute d'avoir vécu de telles expériences, le cœur vidé livré au désespoir? On a compris que l'espace thymique ne donne pas lieu à une localisation mais à une appréhension (un mode de contact) très originaire ${ }^{3}$ de l'espace. Investi par la Stimmung, l'espace résonne maintenant avec l'existence et tous deux vibrent du même ton. Il en découle, pour celui qui en fait l'expérience, le sentiment d'un «accord inconditionnel» entre lui et son environnement. Une telle configuration est porteuse, pour l'homme, de confiance en soi et en son devenir, de confiance en soi et en autrui. Elle possède ainsi les qualités phénoménales pour devenir la base d'appui d'un nouvel élan existentiel qui se concrétise dans un acte de liberté et de responsabilité de soi avec autrui (Lévinas, 1979).

Ainsi, ne dira-t-on plus que l'homme évolue dans un espace à un temps donné mais qu'il meut l'espace et le temps (Von Weizsäcker, 1958). Un espace constitué 
de multiples nuances (espace géométrique, physique, symbolique, technique mais aussi kinesthésique, esthétique, historique, magique, etc.) et que Binswanger a rangées selon les deux catégories de «l'espace orienté» et de «l'espace thymique».

Ces catégories ont été également repérées et commentées par Merleau-Ponty et Foucault qui les ont définies et opposées, respectivement, comme «l'espace clair et objectif» ou «espace du dehors» et «l'espace sombre et anthropologique» ou «espace du dedans». Foucault montre comment l'espace que nous mouvons est fondamentalement hétérogène (orienté et mâtiné de thymique). Le paradigme qui donne toute sa dimension à cette qualité, est celui du voyage de noces. Pour les jeunes filles, explique-t-il, il existait, jusqu'au milieu du $\mathrm{XX}^{\mathrm{e}}$ siècle, cette tradition ancestrale du voyage de noces. La défloration de la jeune fille ne pouvait avoir lieu nulle part et, à ce moment-là, le train ou l'hôtel étaient bien ce «lieu de nulle part», cette hétérotopie sans repères géographiques (Foucault, 1994, 752-762) propice, dirons-nous, à l'accueil du Réel. Foucault identifie en tant qu'espaces anthropologiques d'autres lieux tels que la prison, les cliniques psychiatriques, le cimetière, les jardins, les musées, les bibliothèques, les maisons closes, et les colonies. Loin de s'agir d'une compartimentation de l'espace, la spatialité de ces lieux est thymique car ils auto engendrent une dimension qui leur est propre et qui «parle au cœur» nous dit-il. Chacun a fait l'expérience de se rendre en ces lieux qui ouvrent au silence, au recueillement, à la rêverie, à la descente en soi-même, et simultanément à l'ouverture et à l'accueil de l'alentour, du proche et du prochain. Bien que thymiques, ces espaces sont aussi des espaces historiques qui initient l'individu à sa propre histoire, le centre (le foyer) et l'excentre (les séjours), pour le rattacher à une communauté. C'est dire combien il est essentiel de compter avec la maintenance, le développement ou la restauration de cette qualité d'espace, tant du point de vue de la vie ordinaire que du point de vue de ses avatars psychopathologiques.

Nous partirons d'une problématique de terrain concernant justement la «désorientation» spatiale (le terme doit aussi être entendu, comme souvent en psychologie, dans sa dimension polysémique) manifestée par des personnes traumatisées crâniennes, pour dégager la fonction «d'éclaireur inspiré» qu'assume l'espace thymique pour la conduite humaine. L'atelier «corps et voix » proposé à ces patients se présente comme un lieu de «nulle part» offert au travail de la spatialité thymique de telle sorte que le participant se trouve en disposition d'accueillir, dans l'Ouvert (Maldiney, 2000), le Réel.

\section{L'exemple de l'atelier «corps et voix» avec les personnes traumatisées crâniennes}

\section{Méthodologie de l'action psychothérapeutique}

Problématique et hypothèses intuitives

Notre observation de ces personnes, sur les bases théoriques que nous venons de poser, donne lieu à une interprétation originale de leur « désorientation». Nous partageons pour commencer les remarques des acteurs de l'institution concernant 
ces patients. «Ils sont perdus», «ils sont incapables de se repérer dans l'institution», «celui-là peut rester des heures sur sa chaise sans bouger», «cet autre ne sait pas quel jour on est», «ils n'ont plus de repères dans le temps, pas d'équilibre», «attention, celle-ci peut tomber, elle fait souvent des chutes», «les capacités de celui-là ont régressé depuis quelques mois», «elle a des absences, elle n'est pas là...», «ils n'ont pas de mémoire», constatent quotidiennement les éducateurs. Autant d'énoncés qui témoignent que ces personnes sont effectivement perdues. Mais, en même temps, d'autres énoncés nous laissent penser que quelque chose de l'ordre du Sentir persiste, niché dans la voix ${ }^{4}:$ «Je n'ai plus de mélodie dans ma voix », «ma voix a changé, elle est grave», «je n'arrive pas à articuler comme avant». Ces énoncés dégagent un climat qu'on peut connoter psychologiquement à défaut de l'identifier précisément. Il s'agit d'un climat «d'anticipation inquiète » comme si leurs auteurs exprimaient un «pressentiment» que nous entendons littéralement comme «un sens à venir» se signalant déjà sous la forme vivante d'une «prae sens» (Maldiney, 1973): une présence pour l'instant étrangère à soi. C'est pourquoi nous allons nous demander de quelle perdition s'agit-il réellement au regard des deux qualités hétérogènes d'espace que nous avons posées?

Du point de vue de la spatialité thymique, nous notons que la perdition de ces patients n'aurait pas tout à fait la signification déficitaire qu'elle revêt au regard de la spatialité orientée. Ici, elle nous apparaît comme ouverture à une présence au monde, certes inquiète, par le Sentir. Il nous semble que pour l'instant, c'est par ce fil ténu de la voix altérée (altérité) que les patients tiennent encore discrètement dans l'espace social ${ }^{5}$.

Dans la compréhension phénoménologique, la maladie est moins conçue comme un mode restrictif de rapport au monde que comme un mode palliatif qui sauvegarde l'intégrité de ce rapport (Binswanger, 1998). Pour éclairer ce dernier, notre intervention consistera, dans un premier temps, à observer et à décrire les manifestations présentées par ces patients traumatisés crâniens. Il s'agira de comprendre comment ils s'y prennent pour «tenir (encore) en le monde». Pratiquement, nous reconduirons la dispersion des manifestations ontiques et pathologiques vers les structures existentielles qui la rendent possible. Nous nous ferons ainsi une idée de la «mutation du monde» qui s'est opérée pour ces patients; ce qui reviendra à dégager l'essence même du trouble dont ils souffrent.

Poursuivant notre observation de ces patients, hors atelier, nous nous trouvons en présence de leur «corps en apparition» (Oury, 1990, 115); un corps souvent assis, pris dans une immobilité, peu enclin à initier un mouvement ou un déplacement. Il y a en ces patients quelque chose de resserré, de «pris» dans une pesanteur qui les tire vers le bas. Nous découvrons, à leur contact, un espace thymique lourd, opaque, presque effondré.

Figés, ces patients ont tendance à rester dans l'accrochement aux lieux ou à l'autre. Un accrochement qui semble leur apporter une forme de sécurité qu'ils ne trouvent pas dans le mouvement, le déplacement, l'allant, ni dans les 
représentations qui restent fuyantes. En cela, ils incarneraient la tendance ochnophile élaborée par Balint (Balint, 1972) et reprise par Szondi (Szondi, 1960) (pour l'élaboration $d u$ «facteur $m »$ composant, avec le «facteur $d »$, le vecteur $C \mathrm{du}$ contact) qui forme, ordinairement, avec sa tendance complémentaire de «la marche vers », un mode sensible pré psychologique et tout à fait indispensable de rapport au monde.

Il émane d'eux une atmosphère de profonde tristesse, qui connote psychologiquement l'abattement, l'écrasement, comme si le monde ne s'ouvrait pas à eux mais plutôt les engloutissait, les happait... Ces patients nous donnent l'impression être à côté d'eux-mêmes, comme si leur corps n'était pas tout à fait habité, incarné.

Comme nous l'avons dit, leur corps est souvent en proie au déséquilibre; se tenir debout et marcher ne semble pas aller de soi. Mais plus précisément encore, c'est l'initiation du mouvement, dans le sens de «commencer à», qui paraît problématique. Le «se mouvoir» comme expérience formelle du mouvement, semble atteint dans ses fondements originaires et altéré dans ce que Bin Kimura nomme «schizen- ce qui se fait de et par soi-même» (Kimura, 2000). La corporalité semble touchée au cœur, là où ça «pousse» au mouvement.

De plus, les patients semblent incapables de mouvement spontané. Leur corps est comme réduit à n'être qu'une mécanique aux mouvements purement fonctionnels, incapable de créer ce mouvement existentiel qui relie à la vie et à la dynamique relationnelle. En cela, le patient semble touché dans son rapport au «fond de la Vie» selon la formule de Von Weizsäcker :

«Nous ne vivons que par le maintien d'une relation à ce fond dans le sentir et l'agir. » (Von Weizsäcker, 1958, 23)

Les observations recueillies et les pistes qu'elles soulèvent nous conduisent au constat que c'est toute la dynamique originaire du rapport au monde qui est en panne chez ces personnes. Elles sont figées ou bien se déplacent comme des fantômes. Souvent elles tombent. Toutefois, le fait qu'elles «tiennent encore» en le monde par la voix altérée et cette position pré psychologique d'accrochement nous laisse penser qu'un travail psycho phénoménologique est envisageable. Autrement dit ; notre projet psychothérapeutique visera à travailler avec eux la question du lien originaire — à soi, à autrui, à l'altérité, à l'histoire propre, au corps vécu...

\section{Hypothèse de recherche}

Par le biais de l'atelier «corps-voix », nous proposons à ces patients un espace de «nulle part» conçu de telle sorte que ceux-ci puissent y opérer une conversion du regard, de l'espace clair et objectif du dehors où ils se vivent désorientés et déséquilibrés, vers l'espace sombre et anthropologique du dedans où - c'est le pari psychothérapeutique- ils pourraient vivre l'expérience de se «trouver-créer» une tenue présentielle en le monde et avec autrui. Une tenue cohérente qui manifeste la création d'un rapport proportionné en le monde et avec autrui. 
En effet, si la «marche vers » des patients est entravée, voire rendue impossible dans l'espace orienté, favoriser leur évolution dans l'espace thymique peut leur offrir l'occurrence, par le Sentir et le «se mouvoir», de se constituer une nouvelle unité subjective car une personne est toujours, dans cet espace, préposée à un devenir et ramenée à la certitude de sa présence ; à elle-même et en le monde.

\section{Le dispositif}

Plus précisément, l'atelier «Corps et voix» offre un espace ouvert aux possibles du corps en mouvement et de la voix. Cet espace s'apparente à la «fonction praticable» de J. Oury:

«une délimitation de scène construite et reconstruite en permanence- parce que c'est la scène la plus précaire qui soit[qui] consiste à délimiter un site pour qu'il s'y passe quelque chose.»(Oury, 1990, 116)

Notre dispositif thérapeutique va, par le mouvement et la mise en voix à travers différentes expériences groupales et individuelles, convoquer l'espace thymique dans une activité sans dessein, ludique et créative où l'intention n'est pas de faire mais d'être. Il est question d'y promouvoir la rencontre des «corps en présence». Car la présence d'autrui est effectivement susceptible de faire résonner la présence de celui qui s'y abandonne et de lui faire connaître, par la sensation, la qualité de sa présence propre par laquelle il attestera de son appartenance au monde commun.

L'atelier est mené avec un groupe de 5 personnes $^{6}$. Il se compose de quatre temps sur une durée hebdomadaire d'une heure trente:

1. Le temps du déploiement de la présence corporelle.

2. Le temps de la création (le moment des improvisations dansées).

3. Le temps pour «sortir sa voix» (le moment des propositions vocales et improvisations).

4. Le temps d'élaboration symbolique ce qui est produit à partir des éprouvés corporels vécus (le moment de l'entretien clinique).

\section{L'action psychothérapeutique}

\section{Commencer...}

Le changement de registre (du psychique au pré psychique) recherché s'amorce par un travail de régression qui passe par une désubjectivation des figures de représentation (Leroy-Viémon, 1997). Cette opération conditionne en effet la bascule du regard vers l'espace thymique. Elle doit rencontrer une certaine disposition intérieure des participants: une confiance corporelle par laquelle ceux-ci font crédit au dispositif de pouvoir leur faire, en quelque sorte, «toucher l'intouchable» (nous avons précisé que l'espace thymique n'était pas thématisable mais phénoménisable).

Comment, pratiquement, favoriser cette bascule vers l'espace thymique? 
Parmi les différentes occasions qu'il nous sera donné d'observer au fil des séances, nous rapportons l'exemple suivant. Lors d'un atelier, le jeu fut de projeter le son que le participant émet sur la personne située en face de lui. Projeter le son au-devant de soi sollicite le corps dans son entier: le visage, le regard, la colonne d'air... L'être est appelé quitter son quant-à-soi pour aller, au-devant de soi, vers l'autre.

Lorsque les personnes se placent deux par deux et commencent à produire des sons, il apparait qu'elles n'entretiennent pas vraiment de relation. Le son se perd et retombe vite, les corps sont tendus vers l'arrière, comme retenus par la peur de produire un son et de le recevoir. Cela ne sonne pas. Sachant que, d'une manière générale, l'expérience de la rencontre du visage de l'autre, dans cet «entrelacs» du voyant et du visible (Merleau-Ponty, 1964), appelle autrui en sa présence étrangère et familière à la fois, nous comprenons ce que peut représenter une telle expérience pour des personnes traumatisées crâniennes. On peut penser, en effet, que la problématique de nos patients où le vécu d'étrangeté par rapport à un nouveau corps est si violent, entraîne, en miroir, une identification problématique de la figure de l'autre. Face au regard de cet autre, le «Leib», ce «corps en creux » (Le Poulichet, 2002) associé au sentiment de continuité d'existence n'est pas assez consistant, d'où un sentiment d'effacement ou de persécution face au partenaire.

Comment amener les participants à se confier à l'expérience corporelle et vocale qui devrait favoriser la bascule de l'espace orienté vers l'espace thymique? Comment amorcer la transformation du Körper en Leib comme «pouvoir être»? :

«La chair, le Leib, ce n'est pas une somme de touchers, de sensations tactiles et de kinesthésies, c'est un «je peux».» (Merleau-Ponty cité par Maldiney, 1990)

\section{Désubjectiver les figures pour séjourner avec l'altérité}

Pour favoriser ce mouvement, nous formulons la consigne de projeter le son sur le miroir mural de la salle. Elle conduit les participants à projeter des sons qui, peu à peu, dans l'expiration du souffle, forment une auréole de buée sur le miroir. La buée promeut un écart entre le voyant et le visible, réalisant en quelque sorte la différenciation de la forme et du fond, chère à Maldiney (Maldiney, 1993), qui entraîne la densification du Leib. L'obscurcissement des figures de représentations signe l'avènement de l'espace thymique. La frontière dedans-dehors (Moi-autrui) s'évanouit et les participants sentent résonner leur voix simultanément «en»et «hors» leur corps propre instituant ce dernier en lieu pour l'échange. Lors de la phase d'élaboration symbolique (quatrième temps de la séance), nous pouvons sentir leur voix qui s'élève de manière dense et consistante. Ils sentent que leur voix crée une «cavité intérieure» dans laquelle, par un «retour intérieur» qui s'accomplit dans le climat de recueillement propre à l'espace thymique, ils «séjournent». Dans cet espace, ils rencontrent simultanément l'altérité dans une voix inassignable qui se joint à la leur. Inassignable au sens symbolique, car seul 
compte le grain de la voix, seule compte la valeur thymique de la voix; la valeur symbolique qu'elle peut porter est reléguée au second plan.

Ainsi, la désubjectivation des figures de représentation conduit au Sentir, à l'émergence du Leib comme «corps en creux», à une nouvelle articulation dedans-dehors ainsi qu'à une surprenante densité existentielle: «Hors représentation, j'entends ma voix avec d'autres voix, je sens vibrer la voix, je sens mon souffle, je me sens vivant. » témoigne un participant.

Après ce moment où chacun a pu «séjourner» avec «l'altérité en soi», les participants se replacent dans la configuration initiale: deux par deux, en face à face, pour projeter le son. Nous constatons alors que leur manière d'être avec autrui a changé. Leurs corps se déploient vers l'avant, le son s'est transformé, les voix sonnent et emplissent tout l'espace. Le corps, le visage et le regard sont mobilisés vers l'avant de façon à contacter l'autre, à venir le surprendre et le toucher par la voix vécue maintenant comme prolongement du corps. Ils meuvent le temps et l'espace et l'unité qu'ils constituent est manifestement thymique. De la frayeur, ils sont passés au plaisir confiant de la rencontre avec l'autre.

L'expérience du miroir semble pouvoir garantir au patient qu'il ne sera pas anéanti en s'ouvrant à l'autre par le don de voix. Grâce à elle, le corps cesse d'être vécu comme enveloppe déchue livrée à la dimension persécutrice du regard et de la voix de l'autre et devient Leib, ce corps capable, tout à la fois, d'abriter le Soi (Leroy-Viémon, 2007) et d'accueillir l'autre pour ce qu'il est et également comme un soi-même (Ricœur, 1990). Chacun est désormais relié à autrui depuis une expérience de création et de vérité. En terme de «direction de sens » (Binswanger, 1971 et Chamond, 2004), nous dirons que les patients sont passés d'une direction de sens vers l'arrière à une direction de sens vers l'avant. Leur Dasein (leur présence) se déploie au-devant de soi.

Il semble que faire l'expérience d'éprouver l'altérité en soi (avant d'exister avec autrui) permet, dans un second temps, de pouvoir «trouver une tenue» dans la rencontre avec autrui. Le visage de l'autre renvoie alors le participant à son humanité, à sa possibilité de participer à un monde commun. D'un point de vue atmosphérique (Tellenbach, 1968), le mélange des proches et des lointains est rendu possible à travers cette expérience du touchant-touché. Les présences s'auto engendrent dans un mouvement centrifuge et centripète :

«Ce qui caractérise avant tout le monde humain est qu'un évènement survienne entre deux étants. Le monde de l'entredeux existe sur la crête étroite où moi et toi se rencontrent, audelà de la subjectivité et en deçà de l'objectivité.» (Kimura, 2000)

La relation à autrui, à partir du moment où elle est possible, vient confirmer mon existence en ce monde : 
«Je ne peux éprouver mon corps comme mien et moi-même dans mon existence corporelle que dans une relation Je au monde, dans une délimitation corporelle vis-à-vis de l'altérité, c'est-à-dire dans un rapport avec elle et dans la séparation d'avec elle.» (Straus, 1935, 437)

\section{Déployer l'espace thymique}

Une fois opérée la bascule dans le Sentir, le dispositif, par un travail de la voix, va «pousser» (soutenir) les participants à déployer l'espace thymique, à en goûter la profondeur fondatrice. Les voix vibrent ensemble et donnent une certaine qualité à l'atmosphère de l'atelier qui s'engendre à travers un rythme, un timbre, une tonalité, une intensité; une couleur particulière apportée par les présences de l'instant. Chaque voix recèle sa vie propre et délivre quelque chose de la profondeur de l'être qui la livre: son essence, au-delà de toute forme apprêtée, au-delà de tout détour:

«Je suis un être sonore, mais ma vibration à moi je l'entends du dedans $[\ldots]$ je suis incomparable, ma voix est liée à la masse de ma vie comme ne l'est la voix de personne» (Merleau-Ponty, 1964, 187-188)

Les participants se surprennent à rencontrer leur voix. Ultime passage de l'intériorité vers l'espace extérieur, appartenant à la fois à ces deux espaces, elle est à la fois dans «l'entre» (l'entre-deux moi-autrui) et révèle une présence, un être-là, qui n'est pas moi mais soi-même comme altérité.

«La présence est sa propre possibilité. Elle est «pouvoir être». Perdue, échouée là au milieu de son environnement, elle ne s'y trouve (= n'y est) qu'à s'y trouver (= s'y découvrir en se révélant).» (Maldiney, 1973, 172)

L'intimité (ce qui est caché au-dedans) est donnée en partage et se mélange à d'autres voix, d'autres intimités qui se livrent également. Il est question de descendre avant les mots, de chercher la couleur du son projeté, son rythme et la manière dont ce son entre en résonance dans l'harmonie ou la dissonance avec les autres voix. Faire avec l'altérité, s'y confronter, se joue dans la possibilité de moduler le son émis, de lui donner une forme en formation et de se transformer soi-même, de s'auto engendrer dans la rencontre: il s'agit là d'une véritable autopoièse (Varela, Thompson et Rosch, 1993). L'être y fait l'expérience enthousiasmante et fondamentaIe que, s'il est question pour lui de se transformer dans la rencontre avec le monde et avec autrui, le monde et autrui se transforment également à son contact! Chaque voix convoque l'altérité et provoque son altération (dans le sens de «transformation» — la racine alter nous importe ici). L'être 
sonore éprouve la conviction profonde que le monde est le creuset de son engagement présentiel, corporel. Il le fait vibrer de sa «fréquence», il s'y trouve, y prend pied: il s'y aventure ne craignant plus, désormais, de s'y perdre. La rencontre se densifie dans le mouvement vocal et la personne ne tient dans cette rencontre qu'en s'y risquant complètement. L'être module sa voix au fil des émotions éprouvées dans l'instant:

«J'habite l'espace sonore et en même temps il me traverse [...] cet espace est un espace d'enveloppement et de traversée. » (Maldiney, 1973, 202)

La voix participe de la tenue présentielle de l'être en le monde en donnant corps (Leib) dans l'espace sonore (à la fois orienté et thymique) à une qualité de présence. Elle déploie sa consistance alentour dans une tenue du son qui est appel de l'être à ek-sistere: à sortir de soi pour trouver d'autres voix-voies. Notre sensation, partagée par la plupart des participants, est de baigner dans une sorte de «lumière sonore»; comme le son se diffuse dans l'espace, il diffuse sa lumière particulière. Il remplit l'espace de son volume, il est « volumineux ».

Lorsque le son fait retour vers son émetteur, il est l'écho immédiat et mouvant de son existence hors représentation, (le miroir, lui, renvoie une image sans volume, sans souffle). Le son est à la fois matière tangible et volute invisible émanant d'une voix dans son mouvement d'incarnation. La voix est l'incarnation du son; le son est l'incarnation d'une présence. La voix est un morceau de Réel en soi et le son serait un morceau de Réel de soi donné à entendre. Alors que le Réel est habituellement vécu par la personne traumatisée comme une effraction de l'étrangeté en elle, notre dispositif offre à cette dernière les conditions pour qu'elle se réapproprie sa voix et pour qu'elle en dispose, la module, la fasse jouer dans un nouveau nouage possible «Réel, Symbolique, Imaginaire»; pour qu'elle participe à la création d'une atmosphère par l'inflexion qu'elle porte au groupe. La voix n'est plus vécue comme un objet séparé de soi, comme «objet perdu jamais plus comme avant», comme «objet à articuler péniblement pour se faire comprendre » ou encore comme objet de frayeur car méconnaissable, monstre en soi que l'on n'ose montrer. Elle devient aussi la voie par laquelle le participant accueille la voix de l'autre: l'altérité.

La voix tient le son qui prend le relais de l'habituelle représentation pour une expression cette fois-ci de pure durée (Spüren). Le participant éprouve, par le son égrainé (on pense au grain de la voix de Barthes, 1982, 236-245) son existence propre en sa durée. Il meut le temps dans un espace qui trouve son foyer dans le cœur. Thymique veut dire disposé par une Stimmung qui a son origine dans le thymos en tant que cœur de la possibilisation spatiale et guide de la conduite (Binswanger, 1932). Le participant déploie sa voix là où le cœur lui en dit. La Stimmung donne une direction à cette voix et en guide le déploiement. Puis le retour du son va orienter la disposition et l'intention du participant dans un incessant mouvement d'aller-retour. 
Ainsi P. dira lors d'un atelier: «J'ai chanté et j'ai ressenti ce que je sentais quand j'étais enfant, quelque chose que je n'avais pas senti depuis longtemps. »

Il découvre à ce moment-là un éprouvé qui rétablit le lien avec son enfance, qui le fonde, en deçà et de delà de la dialectique «avant — après l'accident». Nous identifions cet éprouvé comme la tonalité de base de P., sa Stimmung, à la fois toujours là et toujours en formation; sa dynamique propre de transformation de soi. Celle-ci guide sa conduite, le fonde comme être en devenir. En déployant sa voix tout au long des ateliers, P. a (re)trouvé des sensations profondes (existentielles). En paix et en liberté, il a mobilisé en lui-même ses ressources essentielles et visité l'univers de ses possibilités.

Pour nous, le chant se présente comme une possibilité de toucher cette connaissance ontologique de soi-même. Ce déploiement du Dasein fait sentir à l'homme «qui il est» et «d'où il vient». L'homme incarne alors cette spontanéité naturelle qu'ont les enfants et qu'il découvre là comme faisant toujours partie de lui. Au regard du temps ordinaire (chronos), la temporalité de l'espace thymique est d'une autre nature. Elle surgit dans la trame temporelle que forment «passé, présent, futur » pour y inscrire un temps du devenir qui dépasse et englobe le temps historique.

\section{Amplifier le déploiement de l'espace thymique}

Poursuivant nos observations des improvisations vocales en atelier, nous constatons que la prise de risque vocale des participants est limitée lorsqu'ils sont en cercle et qu'ils se voient. Il nous semble que la prégnance de la représentation (l'image de soi, des autres) entrave le lâcher prise. Merleau-Ponty ne nous dit-il pas qu'il y a un narcissisme fondamental de toute vision du fait que, dans le voyant-visible, l'individu se regarde beaucoup lui-même.

Afin de maintenir la continuité de l'expérience thymique, nous transformons la configuration spatiale: le cercle s'ouvre vers l'extérieur de manière à ce que les participants (dont nous sommes) se situent dos à dos, proches les uns des autres. Cette nouvelle configuration nous permet d'échapper ainsi à la prégnance de la vision (de la représentation) et d'accéder au lâcher prise dans un rapport plus senti à ce qui se passe pour se consacrer à un travail du sens des sens dans lequel chacun expériencie (Charbonneau, 2000) la proximité et la vibration (vibrato) du prochain dans la spatialité du Sentir. Nous sommes proches par le sentant, lointains par la représentation. Nous nous adossons sur le son (notre propre son avec celui des autres) alors même que le visage et l'image de l'autre ont disparu. Les sens du Sentir sont tenus en éveil : l'ouïe, comme sens prégnant de l'instant (elle est point d'ancrage et «reliance »); l'odorat, par le fait de respirer les odeurs de chacun; le toucher, par la proximité corporelle que nous entretenons (chaleur des corps et vibrations sonores). Le moment est rythmé par les respirations: mouvement de diastole-systole, d'allers et venues, à l'origine du son et de la Vie.

Dans ce déploiement de l'espace thymique et de la sphère du contact, chaque être sentant entre dans une écoute plus sensible: les voix s'ouvrent, cherchent les 
harmonies, les rythmes, la création d'un chant commun où chacun trouve sa place. Il devient possible de se décaler par rapport à l'autre en chantant dans les silences, ces espaces «entre», pour s'affirmer soi-même. La proximité dans le sentir et la mise entre parenthèse (epokè) du visuel a permis à chacun de se dé-couvrir : faire tomber les voiles de l'image, de la représentation et des enjeux narcissiques pour d'oser se faire entendre. Alors nous constatons que l'écart, créé par la réduction du visuel, devient le lieu d'ouverture à un méta niveau: le principe métanoétique (Kimura, 2000), une direction de sens verticale (Binswanger, 1971, 237-245) qui englobe et déborde l'individu.

Le son en effet se déploie selon une direction centripète vers le haut: il se tourne davantage vers l'altérité comme valeur éprouvée que vers les alter ego, compagnons d'atelier. Il émane d'un foyer sans toutefois mettre encore en tension d'horizon. Nous pensons qu'il vient de s'opérer une nouvelle bascule dans l'espace du dedans. Et plus cet espace est mis en tension et déployé, plus il prend consistance pour chacun, selon un mouvement de charge ou d'auto engendrement (Aulagnier, 1985). Nous décidons de soutenir ce mouvement en modifiant encore la configuration spatiale.

\section{Ouvrir l'accès à la méta rencontre de l'altérité}

Nous modifions la configuration spatiale: les participants sont proches les uns des autres, le visage tourné vers l'intérieur du cercle, ils peuvent se voir et le son est projeté vers le haut. Les modalités d'improvisations et les possibilités de création expérienciées dans la confiance du cercle «dos à dos» permettent de s'exposer, sans risque désormais, au regard de l'autre. La mise en tension de la verticalité continue de se déployer malgré la réintroduction de la représentation: nous y voyons un équilibre réussi du registre de la représentationnel et de celui du Sentir qui co-appréhendent de concert une même situation; comme il est souhaitable théoriquement que cela soit. Le participant jubile que de pouvoir offrir son chant à l'autre.

Le son est projeté vers le haut, «atmosphérisant» l'espace en un dôme (par ailleurs symbole de protection) invisible mais sensible, dont le cercle des participants constitue la base et les souffles unis, la profondeur consentie. Ce dôme est le lieu de nouvelles résonances : les voix sonnent différemment grâce à ce positionnement du visage vers la (même) hauteur. Pour beaucoup de participants qui regardent toujours vers le bas de peur de tomber quand ils marchent, ce moment est vécu comme une expérience d'élévation. Reliés à une base et aspirés vers le haut, ils gagnent en confiance et en dignité.

Les différentes dimensions de l'espace thymique sont explorées: la hauteur dans la formation du dôme, dans l'élévation des voix et des regards ; l'étendue qui se déploie devant eux et, au-delà, qui les dépasse pour ouvrir un horizon; ainsi que la proximité. Ces trois dimensions disposent à l'accueil, en soi, de l'altérité et du monde. Une unité a-thématique est délivrée dans un tel événement. Les participants fabriquent un monde commun dans la création et le partage d'une 
atmosphère et dans le mélange des présences, des proches et des lointains, selon un mouvement centrifuge et centripète : les fonctions attrayantes et distrayantes du sens Oral (Tellenbach, 1968) sont exercées à travers l'échange et le mélange des souffles, des voix, dans un rythme d'inspires et d'expires, d'allers et venues. Par la dislocation ordonnancée des frontières dedans - dehors, l'homme porte audehors son proche et ramène du lointain en lui. La dimension contactuelle de l'existence, telle que l'a décrite Maldiney, y est activée par la pénétration diffuse, le détachement et l'enveloppement; c'est l'expérience du touchant-touché (LeroyViémon, Gal, 2008). La voix et le son de l'un altèrent l'autre, tout comme le son de ce dernier altère le premier, selon un trajet en boucle qui ne se confond pas avec le cercle vicieux de la répétition, puisqu'il progresse de «gradients d'ouverture en gradients d'ouverture» (Maldiney, 1973) vers la hauteur et vers l'étendue (Binswanger, 1947, 237-245).

Chaque participant s'inscrit à la fois dans la continuité et la différence: l'unité dans le cercle ne cesse jamais au-delà des ruptures, des disparitions, des renaissances du son. La cohérence est maintenue, entre les participants et pour chacun, par les voix qui tiennent le son simultanément et en alternance, au rythme des respirations scandées par des silences. Nous y reconnaissons le procès du «cercle de la forme» de Von Weizsäcker :

«[...] en ce qu'il vise la perception et le mouvement dans leur unité et à les comprendre comme couplés dans l'actualité d'une rencontre continuelle du vivant avec le monde [...].» (Kimura, 2000, 49)

Notre rapport au monde est à la fois perception et mouvement, il est intuitif et agissant. Les sensations s'articulent tout naturellement aux représentations dans une «évidence naturelle» (Blankenburg, 1991) et la relation au monde et à l'autre s'en trouve modifiée. Le son se déploie selon une direction centrifuge, il émane d'un foyer et met en tension un horizon qui, pour chacun, rassemble à la fois l'autre, soi et plus que l'autre et que soi, c'est-à-dire le Réel. L'équilibre entre représentation et Sentir porte l'être dans l'Ouvert et lui permet ainsi d'accueillir le Réel. Cet accueil peut alors se réaliser en respect de la nature même du Réel qui est ce que je ne peux connaître mais que je peux maintenant sentir parce qu'il pèse de son juste poids sur mon rapport au monde et à autrui. L'espace thymique joue un rôle déterminant dans cette opération: il doit consister suffisamment pour disposer l'être à la rencontre du monde, de soi et d'autrui.

\section{Incarner le lien avec son prochain}

Nous voulons marquer davantage le circuit des échanges invisibles, le lien entre les participants. Cela nous amène à travailler avec de grands élastiques à rideaux qui relient les personnes entre elles ou à un point fixe de la salle, chacun tenant les élastiques autour des poignets. Le travail de lien à l'autre, de l'être-avec autrui 
dans les propositions dansées, configure un espace thymique invisible et pourtant existant qui vient s'incarner, de manière tangible, à travers l'élastique.

L'élastique incarne la tension entre les participants et la mouvance irreprésentable du groupe que des individus peuvent former. Des lignes élastiques se découpent dans l'espace, des formes s'étirent et se meuvent, se transforment au gré du mouvement groupal. Chaque mouvement a une incidence visible sur l'autre et sur l'espace dessiné. L'appréhension de l'espace se modifie. Un recul panoramique, une vision plus large de la scène et de l'existence se révèlent et offrent l'occasion d'entrevoir le cercle de la vie, l'existence même qui se génère dans ce mouvement hors soi. Comprendre un tel mouvement exprime davantage l'action d'une conscience que la confrontation à une représentation de nous-mêmes.

Les participants reliés par ces élastiques peuvent éprouver physiquement les tensions mises en jeu quand ils dansent. Ils ressentent la présence de chacun et cherchent, par le déplacement, à rééquilibrer ou à déséquilibrer l'ordonnancement des figures produites par leurs corps et les élastiques; ils sculptent leur paysage (Straus, 1935).

L'élastique incarne le lien à autrui par un travail d'aller et venues (vecteur C chez Szondi, 1960). Il conjugue l'approchement et l'éloignement des participants qui jouent des distances entre eux. L'élastique entre en tension à une certaine distance que les participants perdent puis retrouvent et sa tension augmente à mesure que ceux-ci s'éloignent les uns des autres. Le lien à l'autre devient consistant même dans l'éloignement physique dans l'espace orienté où il s'éprouve comme proche ou lointain. Par cet outil, se réalise une convocation de l'invisible qui transforme le rapport proche - lointain et qui met en cause la structure fondamentale de l'espace et du temps.

«Éloigner veut dire faire disparaître le lointain, c'est-à-dire l'éloignement de quelque chose veut dire approche.» (Heidegger cité par Maldiney, 1973, 145)

Partout où il est question de se mouvoir,

«[1]e lointain est la forme spatio-temporelle du Sentir» (Straus, cité par Maldiney, 1973, 144).

L'élastique matérialise, en quelque sorte, le fait que le lointain est une forme de proche. Ce faisant, il intègre, dans l'espace orienté, le mode de relation propre au Sentir et à l'espace thymique. Il réarticule ces deux espaces d'une manière très habitée: l'élastique devient le «fil rouge» grâce auquel les participants peuvent ramener et inscrire l'expérience de la certitude du lien à l'autre dans l'espace social.

«Dans la présence se trouve une tendance essentielle à la proximité.» (Heidegger, cité par Maldiney, 1973, 145) 
Les observations de ces improvisations révèlent un espace thymique riche: les participants sont très mobiles, des possibles s'ouvrent dans leurs mouvements et leur relation à l'autre. Ils trouvent une qualité de jeu, ils rient. Le rire manifeste une confiance et une légèreté d'être qui s'actualise dans la possibilité de rencontrer autrui par le jeu. Les élastiques s'emmêlent, forment des nœuds que les participants défont dans le mouvement même: ils passent dessus, dessous, se rapprochent, s'éloignent, jouent des tensions dans l'élastique pour faire bouger l'autre. Le lien s'incarne dans et par le mouvement.

L'élastique renvoie directement au sujet son tonus présentiel (plus que musculaire) et le mouvement des tensions produites par l'autre. Il rend visible tous les effets de présence mis en jeu. Il convoque de nombreuses directions de sens : étirer, rebondir, éloigner, tendre, détendre, être tenu, retenu, attaché, se suspendre, être suspendu à, etc. Il matérialise, de manière vibratoire, l'atmosphère des présences où chacun est à la fois le réceptacle et l'émetteur de cette vibration. Il présentifie le lien qui fait incessamment retour à la personne, qui modifie son être-là et ce qu'elle va engendrer; un lien qui englobe et dépasse la somme de toutes les personnes réunies et leurs actes conjugués.

L'élastique permet un travail profond sur la modification de la perception des frontières dedans-dehors. Le mouvement se déploie plus loin que le corps propre de la personne. Chacun se prolonge dans le corps de l'autre, par les effets de résonances des tensions à l'œuvre. Chacun (par le mouvement) se fait onde pour se propager dans le corps de l'autre et pour l'appeler à une rencontre où il n'est pas possible de faire semblant. Cette forme d'appel crée du lien. C'est cette création qui renverse «l'engloutissement par le Réel en approche» en «sur-prise d'être soi-même», par l'évènement d'exister. Si les frontières dedans-dehors se réaménagent, c'est pour favoriser l'expérience du prochain: l'appréhension de l'autre comme semblable et différent. Nous comprenons que notre présence peut toucher celui qui se trouve au plus lointain dans le paysage. Nous comprenons que notre être et notre action présentielle se prolongent au-delà de nos limites corporelles psychiques. Notre monde prend ainsi une réalité toute différente: visible et invisible, claire et obscure, lisse et profonde, révélée et secrète, partagée et intime.

\section{l'espace thymique chez le sujet sportif}

L'intérêt que nous portons à l'espace thymique, s'origine dans la compréhension des différentes qualités qui composent l'espace global, trop souvent compris comme une entité indivisible. L'espace thymique est ce «lieu de nulle part» d'où émergent les possibilités intrinsèques de constitution et de transformation de la personne; un lieu d'où se construit le socle des relations avec autrui et avec le monde. L'accès à cet espace nécessite d'opacifier ou d'atmosphériser les représentations pour ouvrir à une qualité d'être au monde qui ne peut être éprouvée, touchée, que dans une expérience vécue qui relève d'une connaissance sans pré requis. Le corps sentant constitue une voie perméable à cette expérience de «toucher l'intouchable»; il s'offre en creux aux expériences de l'être. Nous 
insistons sur le fait qu'il n'est pas d'autre lieu que «le corps vivant et sentant» pour habiter dans son entièreté l'espace sensible de la rencontre humaine.

La valeur opératoire du concept d'espace thymique concerne le champ de la pathologie et le champ de la vie normale.

Du côté de la pathologie, nous avons montré comment le dispositif de l'atelier «corps et voix » permet aux patients traumatisés crâniens de faire l'expérience de l'existence de soi via leurs potentiels corporels et vocaux jusque-là méconnus d'eux-mêmes. Les participants s'y sont découverts «autres » que ce que qu'ils croyaient être du point de vue des représentations (qu'ils se faisaient d'eux-mêmes et que les autres leur renvoyaient). Ils ont pu se dégager d'une définition réductrice «de handicapé » ou «d'invalide» attachée à leur personne. Par la bascule de l'espace orienté vers l'espace thymique, par la densification de ce dernier, par l'ouverture de l'accès à la méta rencontre de l'altérité, par l'incarnation du lien avec son prochain dans un mouvement de retour au monde représenté, notre dispositif a donné de la voix et du souffle au jeu [et au Je] humain (Buytendijk, 1956), du mouvement à l'être qui est porté, en confiance, à expériencier l'auto engendrement de soi en le monde avec autrui.

Du côté de la vie normale, ce dispositif a été testé dans le champ artistique (l'expérience fut menée avec les élèves de l'Ecole Nationale de Cirque de Montpellier). Il a permis aux artistes de trouver de nouveaux outils pour accéder à un état de présence densifiée qui promeut la recherche créative et la création sur scène. Ces ateliers qui visent à développer la créativité rassemblent les conditions nécessaires pour que consistent les liens et l'écoute entre les artistes. Ceux-ci y trouvent de nouveaux appuis et un nouvel élan créatif.

Nous testons également ce travail psychothérapeutique sur l'espace thymique avec des sportifs ${ }^{7}$. La présentation théorique et pratique de l'utilisation de ce concept permet maintenant de formuler quelques pistes de travail qui s'avèrent très pertinentes :

- L'intégration, de manière relativement tranquille ${ }^{8}$, de ce Réel qu'il est coutume (que l'on soit patient, proche ou praticien) d'appréhender du point de vue de sa seule qualité traumatique. La baisse de l'angoisse réalisée grâce à l'atelier offre ainsi une autre manière de vivre le jeu et la compétition: l'adversaire devient le partenaire et le jeu, une œuvre commune que chacun concourt à porter haut.

- La possibilité d'accueillir le Réel et la surprise qu'il contient dans l'ouverture (au lieu d'être «pris» par le Réel) offre une grande disponibilité à la part d'inconnu du jeu. L'imprévisible laisse place à «l'altérité en approche»: le sportif accueille en confiance ce qui n'est pas encore "visible».

- Le travail de «lâcher prise» et de «rééquilibrage» entre les deux registres de la représentation et du Sentir permet au sportif de «se trouver» et de «trouver autrui » davantage par le «sentant» que par le «voir». L'athlète développe alors de nouvelles dispositions dans le jeu ainsi qu'une capacité 
d'adaptation qui ne relève pas de l'anticipation réfléchie mais d'une saisie immédiate et globale de la situation telle qu'elle se présente.

- Par le travail de la profondeur, le paysage situationnel du sportif se transforme: la perception du proche et du lointain se modifie, conférant au lointain le caractère de la proximité, dans le sentir. La distance, considérée du point de vue de l'espace orienté, n'a plus la même valeur: dans le jeu, désormais, l'autre reste proche même dans l'éloignement. Ce sentiment «d'être relié» garantit au sportif que le monde qu'il constitue avec ses coéquipiers, ses adversaires, l'entraîneur, des membres de sa famille et des amis venus le supporter est maintenu dans le mouvement et la durée de l'action sportive. Son lien à (soi et à) autrui perdure, à la fois animé et consistant, fluide et soutenu.

- Appréhender les autres, au sein de l'équipe, par le sentant donne cette possibilité au sportif d'être syntone avec autrui, avec le groupe. L'équipe trouve par là une cohésion qui dépasse les enjeux narcissiques, les rivalités ou les «fraternités» liées aux représentations de l'autre. La cohésion est soutenue par la sensation ou le sentiment de «faire corps» avec l'autre. L'équipe et l'équipe partenaire ou le sportif et son adversaire devenu partenaire, s'unissent pour déployer leurs différentes fonctions dans la perspective d'un jeu commun. L'intégration de chacun dans l'unité semble aller de soi : chacun étant aussi nécessaire et vital à l'ensemble que l'est chaque organe pour le même corps. La cohésion de l'équipe sportive se fonde dans la co-responsabilité de créer le jeu commun, d'être dans le jeu, dans l'instant, plutôt que dans une visée temporelle objectivée qui consisterait à vouloir anéantir l'autre.

- Le sportif peut faire confiance à l'autre, comme à un soi-même. Comme soi-même, l'autre est en capacité de jouer et de déployer l'univers de ses propres possibles. Le crédit que le sportif fait à cet autre est concevable par le seul fait qu'il sent que l'autre ne se réduit pas à la représentation qu'il a de lui : le sportif situe l'autre toujours bien au-delà de ce que ce dernier a déjà montré et bien au-delà aussi de ce dont on peut l'imaginer capable. Par la disposition thymique, le lien d'alliance créé entre les sportifs se manifeste par un don de confiance à l'autre et par le pari en ses compétences. Il est question de se fier à soi, à l'autre et à ce qui advient: voie d'un «enthousiasme» qui laisse au «pouvoir être» la possibilité d'advenir et amène l'autre à porter ce qu'il «peut» avec la plus grande envergure.

Nous espérons avoir montré, à travers ces expériences et recherches, à quel point la prise en compte de l'espace thymique ouvre de nouvelles voies de compréhension de la rencontre humaine. Le sportif y découvre des ressources et des points d'ancrage inédits. Le psychologue y découvre de l'importance qu'il y a à prendre soin de la dimension existentielle de l'être. Mais au-delà de ces deux configurations, il faut comprendre que «l'être homme», par cette profondeur qu'il 
met à jour, préserve l'humanité d'une perdition dans le règne de l'artifice; se confiant à l'espace thymique, il porte haut dans le cœur le goût de la vie. brigitte leroy-viemon,
félicia moscato laboratoire de psychologie expérimentale de la mémoire et de la cognition, EA 3021- université paul-valéry, route de mende 34199 montpellier cedex 05

\section{Notes}

1. Ces recherches ont mis en évidence un mode pré psychique de re-création d'un «monde pour soi avec autrui» qui ouvre comme dès lors une perspective psychothérapique pertinente au regard des pathologies actuelles qui posent la question de la désinsertion et de la désorientation affectives du patient (Leroy-Viémon, Moraguès, Gal, 2002). Plus largement, nous renvoyons le lecteur aux travaux paradigmatiques de Moraguès sur l'état de performance ainsi que les prolongements auxquels ils ont donné lieu pour la psychothérapie des psychoses et du psycho traumatisme. L'originalité et l'efficience de ces dispositifs reposent sur leur capacité de transformer la qualité traumatique de la rencontre avec le Réel en une ouverture confiante à l'altérité (Leroy-Viémon, Gal, 2008 Psychothérapies, $n^{\circ}$ 1, 34-67).

2. Les travaux de J-L Moraguès modélisent ce qu'il en est de cette dynamique (pouvoir «être avec autrui ») dans le champ du sport de haut niveau (Moraguès, 2003).

3. Originaire renvoie au processus originaire élaboré par Aulagnier et conçu par nous (en résonance avec les travaux de l'Ecole Hongroise de psychanalyse) comme un régime de signes essentiellement pré psychique.

4. En posant la voix comme l'un des quatre objets prototypes du Désir (avec les fécès, le sein et le regard), Lacan en fit un «concept frontalier» de la théorie psychanalytique; que nous situons, pour notre part, entre vie psychique et vie pré psychique ou «sensible».

5. Notre dispositif thérapeutique saisira cette occurrence pour entraîner ces patients, par une atmosphérisation provoquée de leur monde propre, à faire l'expérience du Sentir.

6. Deux ateliers de même nature seront mis en place simultanément. Depuis cette expérience, nous proposons régulièrement ce type de travail à des populations différentes : soit dans une visée psychothérapeutique (femmes victimes de violence conjugales, enfants handicapés, trisomiques, hyperactifs); soit dans une visée de développement personnel (artistes de cirque, danseurs).

7. Ces deux dernière années, plusieurs étudiants du Master de psychologie clinique et psychopathologie du sportif ont engagé des recherches dans ce sens. Elles ont fait l'objet de leur mémoire de praticien.

8. Nous observons une baisse de l'angoisse chez les participants au fil des séances.

\section{Bibliographie}

Aulagnier P., 1975, La violence de l'interprétation, Paris, P.U.F.

Balint M., 1972, Les voies de la régression, Paris, Payot.

Binswanger L., 1930, Le rêve et l'existence, in Introduction à l'analyse existentielle, Paris, Minuit, 1971.

Binswanger L.,1932, Le problème de l'espace en psychopathologie, Toulouse, PUM, 1998. 


\section{4}

Filigrane, automne 2008

Binswanger L., 1947, Introduction à l'analyse existentielle, Paris, Éditions de Minuit, 1971.

Blankenburg W., 1971, La perte de l'évidence naturelle, Paris, PUF, 1991.

Buytendijk F.J.J., 1956, A propos du jeu humain, L'évolution psychiatrique, 1, 63-67.

Chamond J. (Ed). 2004, Binswanger et les directions de sens, in Les directions de sens, Paris, Le Cercle Herméneutique, 19-42.

Charbonneau G., 2000, De la Nostrité, Aspects phénoménologiques et psychopathologiques de l'expérience du Nous, in Trois pensées du comprendre: Bernard Groethuysen, Wolfgang Blankenburg, Jacques Garelli, Paris, Le Cercle Herméneutique, 134-148.

Foucault M., 1994, Dits et écrits, Paris, Gallimard, 4 volumes. Defert D. et Ewald F. Ed,.

Gros C., 1998, Préface, in Le problème de l'espace en psychopathologie, Toulouse, Le Mirail.

Heidegger M., 1927, L'être et le temps, Paris, Gallimard.

Jonckheere P., 1998, Passage à l'acte, Bruxelles, De Boeck-Wesmael.

Kimura Bin., 1988, L'Entre, Grenoble, Millon, 2000.

Le Poulichet S., Psychanalyse de l'informe, Paris, Aubier, 2002, 56.

Leroy-Viémon B., 1997, L'altérité fondatrice, Paris, L'Harmattan.

Leroy-Viémon, B. Trouillet, R. Bruère-Dawson C.G., 2006, La notion de représentation en psychologie clinique, in Blanc N. (Éd.), Le concept de représentation en psychologie, Paris, In Press Editions, 43-89.

Leroy-Viémon B., 2007, Le genou de Clémence ou l'espace nostrique comme perspective psychothérapeutique, in Pratiques psychologiques, Paris, Elsevier, 267-281.

Leroy-Viémon B., Gal C., 2008, Utilisation du contact comme ouverture à la rencontre. L'exemple du psychodrame existentiel pour la psychothérapie des personnes psychotiques. Psychothérapies, n 1 , 34-67.

Lévinas E., 1979, Le temps et l'autre, Montpellier, Fata Morgana.

Maldiney H., 1954. Regard, espace, parole, Lausanne, L'Âge d'Homme, 1973.

Maldiney H. 1990, La dimension du contact au regard du vivant et de l'existant, in Schotte J. (Ed). Le contact, Bruxelles, De Boeck-Wesmael.

Maldiney H., 1993, Art, folie, thérapie, essais de conceptualisation, in Actes du colloque des 18 et 19 novembre. Les murs d'Aurelle, Montpellier.

Merleau-Ponty M., 1960, Le visible et l'invisible, Paris, Gallimard.

Moraguès J-L., 2003, Psychologie de la performance, Corps motionnel, corps pulsionnel, Montpellier, UM3.

Oury J., 1990, Pathique et fonction d'accueil en psychothérapie institutionnelle, in Schotte J. (Éd), Le contact, Bruxelles, De Boeck-Wesmael, 111-128.

Ricoeur P., 1990, Soi-même comme un autre.

Sauverzac (de) J-F., 2001, Sur les origines anthropologiques du réel chez Lacan, in Cliniques méditerranéennes, $\mathrm{n}^{\circ} 63$ $-2001 / 1,223-237$.

Straus E., 1935, Du sens des sens, Grenoble, Millon.

Szondi L., 1960, Lehrbuch der experimentellen Triebdiagnostik, Bern, H. Huber, (2éd.).

Tellenbach H., 1968, Goût et atmosphère, Paris, P.U.F.

Varela F, Thompson E. et Rosch E., 1993, L'inscription corporelle de l'esprit, Paris, Seuil.

Weizsaecker, V. V. 1958, Le cycle et la structure, Paris, Desclée de Brouwer. 